\title{
Le conditionnel en français : énonciation, ultériorité dans le passé et valeurs modales
}

\author{
J. Bres, S. Azzopardi, S. Sarrazin*
}

Comme on le sait, le paradigme morphologique appelé conditionnel en français est issu, dans la plupart des langues romanes, de la grammaticalisation de la périphrase bas-latine : V. inf. + habere à l'imparfait (catalan, espagnol, français, italien, occitan) ou au prétérit (italien). Cette grammaticalisation est très avancée en français dès les premières attestations ${ }^{1}$ puisque le conditionnel se présente sous une forme synthétique : l'auxiliaire initial latin habebat, décatégorisé et érodé, est devenu un affixe verbal : cantare habebat > chanteroit. La forme, qui a dès le départ une valeur temporelle d'ultérieur du passé ${ }^{2}$, a développé des emplois modaux nombreux, que Dendale (2001 : 9) classe en trois ensembles : éventualité, emprunt, atténuation. Quel lien entre la valeur temporelle d'ultériorité dans le passé et ces emplois modaux ? Nous faisons l'hypothèse que ceux-ci sont dérivés de la valeur temporelle du conditionnel, valeur qui procède directement des constituants morphologiques $-r$ - et $-a t^{3}$.

Dans un premier temps nous établirons, à partir de ces bases morphologiques, la spécificité énonciative de la valeur temporelle du conditionnel. Nous décrirons ensuite les chemins qui conduisent du temporel au modal à partir de l'étude d'emplois représentatifs des trois ensembles modaux précités. Compte tenu de l'espace dont nous disposons, nous ne pourrons qu'esquisser l'analyse, que nous conduirons sur la seule forme simple du conditionnel (conditionnel présent) en français.

1. LE CONDITIONNEL EN EMPLOI TEMPOREL: UN ULTERIEUR DU PASSE SUBJECTIF

\footnotetext{
* Praxiling, UMR $5267 \quad$ CNRS-Montpellier III, jacques.bres@univ-montp3.fr' azzo.soph@gmail.com'sophie.sarrazin@univ-montp3.fr empedementz / Qu'elle perdesse sa virginitet ('Eulalie se raidit : elle souffrirait la torture plutôt que de perdre sa virginité'). 2 Et peut-être aussi une valeur modale d’hypothèse. Pour le débat non résolu : le conditionnel, temps et / ou mode ?, cf. i. a. Fleischman $1982: 64$

3 Par convention, nous adopterons, pour la notation de ce morphème commun au conditionnel et à l'imparfait de l'indicatif, la désinence de 3ème personne.
} 
Le conditionnel est morphologiquement composé des deux affixes - $r$ - et -ait, d'où procède sa valeur temporelle d'ultérieur du PASSÉ (entre autres : Reichenbach 1947, Gosselin 2001) : l'affixe -ait pose dans le PASSÉ un élément à partir duquel le procès est situé en ultériorité par l'affixe - $r$-, comme dans Pierre a dit que Corinne reviendrait. Nous avons analysé (Bres 2009) cet élément non comme un simple point de repère (Reference Point, Reichenbach 1947), mais comme un autre acte d'énonciation (e), réalisé par un énonciateur $\mathrm{e}_{1}$, antérieur à l'acte d'énonciation principal (E) et rapporté par son énonciateur $E_{1}^{4}$. C'est à partir de cet acte énonciatif (e) que le procès est construit en ultériorité anaphoriquement, ce qui rend compte de ce qu'il puisse référer à un événement antérieur, contemporain ou postérieur au nunc : Pierre a dit que Corinne reviendrait la semaine dernière / aujourd'hui / la semaine prochaine.

La structure temporelle du conditionnel en tant qu'ultérieur du PASSÉ est à l'origine d'un fonctionnement énonciatif dialogique : du fait de l'interaction des deux affixes - $r$ - et -ait qui le composent, ce temps double systématiquement l'énonciation principale (E) d'une énonciation rapportée (e), explicite (discours indirect), présupposée ou sous-entendue (discours indirect libre notamment : Pierre souriait : oui, Corinne reviendrait). Il ne peut actualiser le sens temporel d’ultériorité que dans le cadre syntactico-énonciatif du discours rapporté.

On dira donc que le conditionnel temporel est un ultérieur du passé subjectif, valeur qui procède des éléments morphologiques qui le composent, et que l'on peut représenter de la sorte : $[(\mathrm{e})<(\mathrm{E}) ;(\mathrm{e}) \rightarrow \mathrm{P}]$, ce qui signifie que son emploi implique une énonciation rapportée (e) antérieure à (E) (affixe -ait) ; et que le procès au conditionnel $\mathrm{P}$ se construit anaphoriquement comme ultérieur à l'énonciation (e) (affixe -r-).

\section{DE L'EMPLOI TEMPOREL AUX EMPLOIS MODAUX}

Que reste-t-il des deux éléments que nous avons posés à la base de la structure du conditionnel-temps dans les conditionnels dits d'emprunt (1) et d'atténuation (2) que nous prendrons ici comme exemples d'emplois modaux?

(1) Le recours aux biocarburants serait un remède pire que le mal (titre, Le Monde, 20 juillet 2011)

(2) Réservation téléphonique (2009) dans un restaurant :

- le Duo bonsoir

- bonsoir je voudrais réserver deux places pour ce soir

- oui ce serait à quel nom ?

$4 \quad$ E1et e1 peuvent être en relation de coréférence (je disais que Corinne reviendrait) ou non (ex. cité supra). 
Apparemment rien. Ni énonciation rapportée passée (e), ni ultériorité anaphorique: les procès signifiés par être ne semblent pas construits comme ultérieurs anaphoriquement à partir d'un acte d'énonciation (e) antérieur à (E) ; mais comme (temporellement) simultanés à (E) (plus précisément même ici, comme inclus dans sa temporalité). En (2) p. ex., on peut, à l'effet d'atténuation près, remplacer le conditionnel ce serait par le présent : " c'est à quel nom ? ». Et pourtant... Notre hypothèse est que ces emplois sont dérivés de la structure temporalo-énonciative décrite supra en 1., dérivation que nous fondons sur le fait qu'elle persiste, de façon abstraite, dans les effets de sens que le conditionnel produit en interaction avec le contexte dans ces différents tours. Nous allons nous attacher à expliciter cette rémanence et à expliquer la dérivation qui permet de passer des deux éléments de la structure temporelle $[(\mathrm{e})<(\mathrm{E}) ;(\mathrm{e}) \rightarrow \mathrm{P}]$ aux effets de sens modaux produits en (1-2), qui relèvent des deux catégories du médiatif (Lazard 1956, Guentcheva 1996 ; correspondant à l'evidentiality en anglais) et de l'épistémique.

\subsection{De l'énonciation antérieure rapportée $((e)<(E))$ à l'effet de sens médiatif}

Reprenons l'occurrence de conditionnel dit d'emprunt :

(1) Le recours aux biocarburants serait un remède pire que le mal (titre, Le Monde, 20 juillet 2011)

interprétable comme le fait que $\mathrm{E}_{1}$ (correspondant au journaliste) impute cet énoncé à un énonciateur antérieur $\mathrm{e}_{1}$ (non précisé) (et secondairement par inférence suspend son engagement épistémique quant à la validité de l'explication proposée).

Le chemin qui mène de l'effet de sens temporel à l'effet de sens d'emprunt peut être décrit comme le passage d'une structure syntaxique de discours rapportant un acte d'énonciation antérieur (e) à une structure qui, par abstraction, le présuppose sans rien en dire, ou en explicitant au mieux son énonciateur dans une forme de modalisation en discours second en selon / d'après $x$ (selon certains experts, le recours (...)). L'acte d'énonciation (e) n'est donc pas effacé, mais persiste sous une forme allégée, de moindre intension et de plus grande extension : celle d'une instruction (donnée par le conditionnel) de dédoublement énonciatif dans l'antériorité de (E). Ce qui était une condition d'emploi du conditionnel temporel - l'ancrage à un acte d'énonciation antérieur (e) s'incorpore à son sémantisme, et est à l'origine de l'effet de sens d'emprunt : en (1), c'est le conditionnel et lui seul qui marque l'énoncé comme médiatif: il signale que le jugement selon lequel «le recours aux carburants est un remède pire que le mal » n'est pas le fait de $E_{1}$, mais procède d'une source énonciative antérieure (e1) non coréférentielle.

Notre hypothèse permet, entre autres, d'expliquer le fait suivant: au conditionnel, la modalité médiative correspond à de l'ouï-dire, qui présuppose un 
acte d'énonciation antérieur, et jamais aux autres structures médiatives possibles : folklore, savoir encyclopédique, inférence, qui n’ont pas cette présupposition $^{5}$.

On peut pareillement rendre compte de l'emploi d'atténuation à partir de la présupposition d'un acte d'énonciation implicite antérieur, même s'il a moins d’évidence. Reprenons (2) :

(2) - bonsoir je voudrais réserver deux places pour ce soir - oui ce serait à quel nom?

Le présent «c'est à quel nom ? » imputerait l'acte de question sur l'identité, potentiellement menaçant pour la face de l'interlocuteur, au locuteur-énonciateur $E_{1}$. Le conditionnel, en présupposant fictivement un $e_{1}$ antérieur, ici coréférent à $\mathrm{E}_{1}$, introduit une distance temporelle énonciative, ce qui, en interaction avec le contexte, revient à bémoliser l'acte interrogatif, et produit le sens de politesse comme ménagement du territoire de l'interlocuteur: la distance énonciative comme signifiant de la distance interlocutive.

Par sa capacité à poser un énonciateur $\mathrm{e}_{1}$ antérieur à E1, le conditionnel a donc pu développer des effets de sens relevant de la modalité médiative comme l'emprunt (dans lequel $\mathrm{e}_{1}$ ne coréfère pas à $\mathrm{E} 1$ ) ou comme l'atténuation (dans lequel $\mathrm{e}_{1}$ coréfère à E1).

\subsection{De la modalité médiative à une forme particulière de modalité épistémique : la suspension du jugement}

La rémanence de l'énonciation rapportée sous forme de présupposition d'un énonciateur antérieur nous semble fortement validée par le chemin qui conduit du médiatif à l'épistémique. L'énoncé d'emprunt (1) semble associer à la médiation énonciative la valeur épistémique d'incertitude. Regardons-y cependant de plus près: plutôt qu'à un jugement d'incertitude, le travail d'abstraction de l'énonciation (e) conduit d'abord, au delà du médiatif, à suspendre le jugement épistémique de $\mathrm{E}_{1}$, ce qui, par inférence, produit l'effet de sens d'incertitude.

On répartit d'ordinaire les emplois modaux du conditionnel en deux groupes : médiatifs, épistémiques. De fait, les différents tours amalgament les deux éléments de la présupposition d'un $\mathrm{e}_{1}$ antérieur et de la suspension du jugement épistémique, dans des proportions différentes. Tendanciellement, le premier élément est plus saillant dans les emplois dits médiatifs, le second dans les

5 L'emploi conjectural du conditionnel - «Elle ne put résister au torrent de bonheur qui inondait son âme après tant de jours de désespoir. Aurais-je de l'amour pour Julien, se dit-elle enfin? (Stendhal, Le Rouge et le noir) - ne contredit pas notre explication (Bres et Azzopardi 2012). 
emplois dits épistémiques. Mais aucun n'est pur de l'alliance des deux. Par exemple nous avons expliqué l'effet d'atténuation de (2) par l'imputation fictive de l'énoncé à un $\mathrm{e}_{1}$ antérieur. Ledit effet est également produit par ce qu'induit cette imputation: que le locuteur suspend son jugement, c'est-à-dire, en interaction avec le contexte, qu'il se place poliment en retrait: le tour est médiatif et épistémique.

Nous avons explicité le chemin d'abstraction qui conduit d'une énonciation antérieure rapportée $[(\mathrm{e})<(\mathrm{E})]$ aux effets de sens médiatif et épistémique. Qu'en est-il du second élément, tout aussi structurant, l’ultériorité du procès ?

\subsection{De l'ultériorité du procès $[(e) \rightarrow P J$ à la modalité épistémique de l'éventualité}

Dans les emplois modaux, le procès au conditionnel ne peut plus se construire en ultériorité anaphorique dans la mesure où l'acte d'énonciation rapporté (e) n’est plus posé. Qu'advient-il de cet élément de base du conditionnel-temps ? Nous faisons l'hypothèse qu'il emprunte le chemin qui, dans de nombreuses langues (Heine 2003 : 594), conduit du sémantisme concret de l'ultériorité à un élément de sens plus abstrait : la modalité épistémique de l'éventualité pour reprendre la classification de Dendale (2001).

L’ultériorité, - au moins dans la culture occidentale - qu'elle soit déictique (futur) ou anaphorique (conditionnel), est phénoménologiquement liée à une représentation ramifiée du temps (Gardies 1975, Martin 1981): alors que le passé comme le présent sont unilinéaires, l'ultérieur est le champ des possibles. En emploi temporel (Pierre a dit que Corinne reviendrait), l'ultériorité anaphorique implique l'incertitude de la réalisation effective du procès. Ce qui n'était qu'une inférence dans le conditionnel-temps devient un élément saillant dans les emplois modaux. En (1), le jugement selon lequel le recours aux biocarburants serait un remède pire que le mal est présenté non comme réel mais comme éventuel ; en (2), le conditionnel " éventualise » l'acte de question alors même que le locuteur est en train de le réaliser intonativement, ce qui concourt à l'effet de sens d'atténuation.

Par des chemins différents, les deux éléments structurants de la morphologie du conditionnel (-ait, -r-) en viennent à signifier la modalité épistémique, dans des aspects complémentaires: la suspension du jugement (avec inférence selon laquelle si $E_{1}$ ne se prononce pas, c'est qu'il place le procès dans l'incertitude), et le positionnement dans le champ des possibles. Il va de soi que ce n'est que pour l'analyse que nous avons séparé les chemins modaux des deux éléments : dans les faits, il y a interaction entre les processus qui affectent chacun d'eux.

Généralisons : l'ensemble des effets de sens modaux du conditionnel est produit par les trois ingrédients de la distance énonciative, de la suspension du jugement, et de l'éventualité, dérivés de la structure temporelle, en interaction avec des contextes différents. Ce que nous illustrerons rapidement par un $3^{\text {ème }}$ type d'emploi : le conditionnel dit (pré)ludique : 
(3) (deux enfants jouent aux vendanges avec des playmobils)

- $\quad$ alors moi je prendrais le tracteur et j'irais à la cave coopérative pendant que toi tu couperais des raisins

Cet emploi apparaît toujours dans un contexte de jeu (d'enfants la plupart du temps), qui implique les partenaires de l'interlocution s'inventant une autre identité, à partir de laquelle ils développent des activités sur le mode du faire semblant. Le conditionnel participe activement à la production de l'effet (pré)ludique en ce qu'il permet la dissociation énonciative : il présuppose un énonciateur $\mathrm{e}_{1}$ pour qui "prendre le tracteur " et "aller à la cave " sont des possibles, alors que $\mathrm{E}_{1}$ sait très bien qu'ils ne correspondent pas à la réalité effective. Cependant $E_{1}$ suspend son jugement épistémique, ce qui permet à l'espace transitionnel du jeu de se développer dans le champ des possibles. Notons que les auxiliaires modaux épistémiques (en fr. devoir, esp. deber, anglais can, must, may), qui trouvent leur origine dans des modalités de l'agent, ne sauraient participer à la production du sens (pré)ludique : ils ne présupposent pas de dédoublement énonciatif.

\section{CONCLUSION}

Le français, comme la plupart des langues romanes, a développé, à partir des éléments morphologiques $(-r$-, - ait) structurant l'emploi temporel du conditionnel, des emplois modaux médiativo-épistémiques. Nous avons parlé, dans notre étude, de dérivation et utilisé le terme imagé de chemin, emprunté à l'approche de la grammaticalisation (i. a. Bybee et al., 1994). S'agit-il là d'un processus relevant de la grammaticalisation ? Nous réservons notre réponse pour une recherche ultérieure, nous contentant pour l’heure de préciser trois points :

- Le parcours de ce chemin n'a rien d'obligatoire. Pour ne prendre comme exemples que l'espagnol et le français : ces deux langues, si elles ont dérivé des valeurs modales à partir de la valeur temporelle de manière globalement semblable, l'ont fait parfois différemment: p. ex. l'espagnol ne peut produire l'effet de sens (pré)ludique avec le conditionnel ; le français, de son côté, n’a pas développé, à partir de ce temps, l'effet de sens conjectural fondé sur un raisonnement déductif (Azzopardi 2011, Sarrazin 2012) :

(3) Eugenio se volvió a casar al poco, con una chica muy joven, ni veinte años tendría, y menos que aparentaba. (Lucía Etxebarria, Una historia de amor como otra cualquiera, 2004)

(3a) Eugène se remaria rapidement, avec une fille très jeune, elle *aurait / devait même pas avoir 20 ans (...). 
- Le cheminement semble être diachronique: si nous ne disposons pas de datation pour le tour (pré)ludique, il est désormais établi que l'emploi d'emprunt dont les prémisses remontent au XVI ${ }^{\mathrm{e}}$. (Bourova et Dendale 2009) se développe au $\mathrm{XIX}^{\mathrm{e}}$ et surtout au $\mathrm{XX}^{\mathrm{e}}$; et que celui d'atténuation apparaît dans les soties du $\mathrm{XV}^{\mathrm{e}}-\mathrm{XVI}^{\mathrm{e}}$. (Wilmet 1970 : 412) (cf. ici même l'article de Patard et De Mulder).

- La productivité du conditionnel roman ne se limite pas à la dérivation de valeurs modales. Dans la plupart des langues de cette famille, s'est développée au $\mathrm{XIX}^{\mathrm{e}}$, à partir de la valeur temporelle d'ultérieur du passé subjective, une valeur temporelle d'ultérieur du passé objective (Damourette et Pichon 1936, Bres 2012) :

(4) Elle monta, posant la sac à la place de ses pieds. Durant tout le trajet le sac gênerait ses pieds mais Kastner n’oserait pas suggérer qu'on le tranférât sur la banquette arrière. (Echenoz, Les Grandes blondes, 1995).

\section{BIBLIOGRAPHIE}

Azzopardi S. 2011, Le futur et le conditionnel : valeur en langue et effets de sens en discours. Analyse contrastive espagnol / français, Thèse de doctorat, Université Paul-Valéry Montpellier III.

Bourova V. et Dendale P., 2012, "Serait-ce un conditionnel de conjecture? Datation, évolution et mise en relation des deux conditionnels à valeur évidentielle », Cahiers Chronos, à paraître.

Bres J., 2009, "Dialogisme et temps verbaux de l'indicatif », Langue Française $163,21-39$.

Bres J. 2012, «L'expression verbo-temporelle de l'ultériorité dans le PASSE : de la subjectivité à l'objectivité ", CMLF 2012, www.ilf.cnrs.fr

Bres J. et Azzopardi S., 2012, "On aurait oublié les clés du dialogisme sur la porte de l'analyse? De l'effet de sens de conjecture du futur et du conditionnel en français ", in Bres J., Nowakowska A., Sarale J.-M., Sarrazin S., (éd.), Dialogisme : langue, discours, Bruxelles : Peter Lang, sous presse.

Bybee J., Perkins R. Et Pagliuca W. 1994, The evolution of grammar: tense, aspect, and modality in the languages of the world, Chicago-Londres: The University of Chicago Press.

Damourette, J. \& Pichon, J. (1911-1936) Des mots à la pensée: Essai de grammaire de la langue française (Tome 5). Paris: D’Artrey.

Dendale P., 2001, «Les problèmes linguistiques du conditionnel français », in Dendale P. et Tasmowski L. (éds), Le conditionnel en français, Université de Metz : Recherches linguistiques 25, 7-18.

Gardies J.-L., 1975, La logique du temps, Paris : PUF.

Guentcheva Z., 1996, (éd.), L'énonciation médiatisée, Louvain : Peeters.

Lazard G., 1956, "Caractères distinctifs de la langue tadjik », Bulletin de la Société de linguistique de Paris, 52, 117-186.

Gosselin L., 2001, "Relations temporelles et modales dans le conditionnel journalistique », in P. Dendale et L. Tasmowski 2001, 45-66. 
Heine B. 2003, « Grammaticalization », in B. Joseph et R. Janda, The Handbook of Historical Linguistics, Oxford: Blackwell, 575-601.

Martin R., 1981, "Le futur linguistique : temps linéaire ou temps ramifié ? (à propos du futur et du conditionnel en français) », Langages 64, 81-92.

Reichenbach H., 1947, Elements of symbolic logic, New York, Dover Publications, 1980.

Sarrazin S., 2012, «Dialogisme de langue et dialogisme de discours: des emplois dits concessifs du futur et du conditionnel en espagnol », in J. Bres, A. Nowakowska, J.-M.Sarale, S. Sarrazin (éds), Dialogisme : langue, discours, Berne : Peter Lang., à paraître.

Wilmet M., 1970, Le système de l'indicatif en moyen français, Genève, Droz. 